\title{
Research Square \\ High-resolution solar image reconstruction based on non-rigid alignment
}

\section{Hui Liu ( $\square$ liuhui@ynao.ac.cn )}

Yunnan Astronomical Observatory: Chinese Academy of Sciences Yunnan Observatory https://orcid.org/0000-0003-2714-6811

\section{Zhenyu Jin}

Yunnan Astronomical Observatory: Chinese Academy of Sciences Yunnan Observatory

\section{Yongyuan Xiang}

Yunnan Astronomical Observatory: Chinese Academy of Sciences Yunnan Observatory

\section{Kaifan Ji}

Yunnan Astronomical Observatory: Chinese Academy of Sciences Yunnan Observatory

\section{Research Article}

Keywords: High resolution reconstruction, Speckle masking, Speckle interferometry, Shift-Add, NVST

Posted Date: February 2nd, 2022

DOI: https://doi.org/10.21203/rs.3.rs-1275663/v2

License: (c) (1) This work is licensed under a Creative Commons Attribution 4.0 International License. Read Full License 


\title{
High-resolution solar image reconstruction based on non-rigid alignment
}

\author{
Hui Liu ${ }^{1}$ (D) Z Zhenyu $\operatorname{Jin}^{1}$ (D) . \\ Yongyuan Xiang ${ }^{1}$ (D) Kaifan $\mathbf{J i}^{1}$ (D)
}

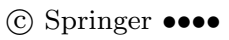

\begin{abstract}
Suppressing the interference of atmospheric turbulence and obtaining observational data with high spatial resolution is an important problem to be solved in ground observation. One way to solve this problem is to perform statistical reconstruction of short-exposure speckle images. Combining the advantages of the classic Shift-Add and speckle masking algorithms, this paper proposes a novel reconstruction algorithm-NASIR (Non-rigid Alignment based Solar Image Reconstruction). NASIR performs pixel-by-pixel non-rigid distortion correction on speckle images by establishing a computational model between geometric distortion and intensity distribution, thereby reconstructing the phase of the object image at each frequency. We compared the reconstruction performance of speckle masking and NASIR in terms of correlation coefficient, power spectrum and intensity profile variation coefficient through reconstruction experiments of NVST (1m New Vacuum Solar Telescope) observational data. The results show that when seeing is good $\left(\boldsymbol{R}_{0}>10 \mathrm{~cm}\right)$, both speckle masking and NASIR can achieve better reconstruction results. When seeing decreases $\left(\boldsymbol{R}_{0}<5 \mathrm{~cm}\right)$, the noise will exacerbate the phase recursion error of speckle masking, resulting in the generation of spurious structures, while NASIR recovers the phase by spatial alignment, which can avoid error accumulation. Moreover, NASIR performs speckle interferometry on the aligned speckle images, which further improves the reconstruction quality of the modulus, so its reconstruction results have a higher cutoff frequency and more significant structural contrast. In addition,
\end{abstract}

$\triangle$ K.F. Ji

jkf@ynao.ac.cn

H. Liu

liuhui@ynao.ac.cn

Z.Y. Jin

kim@ynao.ac.cn

Y.Y. Xiang

xiangyy@ynao.ac.cn

1 Yunnan Observatories, Chinese Academy of Sciences, Kunming, Yunnan 650216,

Peoples Republic of China. 
NASIR reconstruct the entire field of view in parallel at one time, without phase recursion and block-by-block reconstruction, so its computation time is only about $50 \%$ of that of speckle masking. Therefore, we consider NASIR to be a more robust and efficient method that combines the respective advantages of spatial domain reconstruction and frequency domain reconstruction. It is believed that the application and further research of NASIR will promote the development of ground-based observation data processing technology.

Keywords: High resolution reconstruction, Speckle masking, Speckle interferometry, Shift-Add; NVST

\section{Introduction}

At present, the research of solar physics has entered the era of small scale and fine structure, and high-resolution observational images are urgently needed. However, diffraction-limited imaging cannot be achieved with ground-based optical telescopes due to the interference of the turbulent atmosphere.

To mitigate the adverse effects of the atmosphere and achieve near-diffractionlimited imaging, the researchers propose a speckle imaging method for highresolution reconstruction by statistical analysis. The speckle imaging method started from the pioneering work of Labeyrie (Labeyrie, 1970), and its processing object is the speckle images. Speckle images are short-exposure images taken with a telescope with an aperture much larger than the atmospheric coherence length. The speckle imaging method can obtain high-resolution reconstructed images close to the diffraction limit of the telescope, and has formed a relatively complete theoretical support.

Speckle imaging methods can be roughly divided into spatial domain and frequency domain reconstruction methods. Spatial reconstruction methods directly reconstructs the spatial structure and intensity distribution of the object. This type of methods are characterized by their simple algorithm and clear spatial description. The typical representative of the spatial domain reconstruction method is Shift-Add (Bates and Cady, 1980; Qiu et al., 2001). Frequency domain reconstruction methods generally reconstruct the phase and modulus separately, the phase is reconstructed by speckle masking (Lohmann, Weigelt, and Wirnitzer, 1983), and the reconstruction of the modulus usually uses speckle interferometry (Labeyrie, 1970). Most of the above-mentioned classic algorithms originated in the 1970s and 1980s, and subsequent researchers also have a lot of research work to effectively improve these methods (Jin, Lin, and Liu, 2008; yuan Xiang, Liu, and yu Jin, 2016; Huo and Zhou, 2010; Liu et al., 1999). However, there are still issues that need to be further studied and resolved in practical applications.

The key issue of spatial domain methods is how to accurately align the regions containing object diffraction imaging information. Taking Shift-Add as an example, the algorithm is based on the assumption that the maximum intensity points of the speckle images coincides with the maximum intensity point of the object image, and performs rigid translation (uniform displacement of the entire image) and superimposition of each speckle images to obtain a reconstructed 
image. However, for an extended source like the Sun, due to the influence of the surrounding structure, the distribution of maximum intensity points will change greatly under the action of the atmospheric. Not only will there be multiple points of maximum intensity, but their positions will also deviate. This makes the assumption that the points of maximum intensity coincide no longer holds. Furthermore, the overall shift and add of the image ignores the difference in displacement and distortion caused by atmospheric turbulence to different parts of the field of view, which makes it difficult to obtain satisfactory reconstruction results with the traditional rigid Shift-Add.

The frequency domain reconstruction algorithm for speckle imaging is currently the most widely used method for high-resolution reconstruction of solar observation data. The most important ground-based solar telescopes in the world, such as NVST (Liu et al., 2014) and GST (Goode Solar Telescope) (Cao et al., 2010), use frequency-domain statistical reconstruction methods (Liu et al., 1999). A typical representative of the frequency domain reconstruction methods is speckle masking.

Since the speckle masking method estimates the phase of the object based on the triple correlation of the speckle images, that is, recursively from the lowfrequency phase to the high-frequency phase, it is inevitably affected by noise such as photon noise during the recursion process (Liu et al., 1999). Due to the recursion, the noise influence will accumulate in the high frequency part, and in severe cases, it will obviously distort the fine structure of the object. Therefore, when the seeing is poor, the reconstruction resolution of speckle masking will be significantly reduced. In the future, the aperture of the solar telescope will reach $6 \sim 8$ meters, so the phase recursive path for speckle masking will increase proportionally with the aperture, and the cumulative error of the recursion will be more serious; In addition, the triple correlation statistics are limited by the isoplanatic region. It is necessary to segment and reconstruct the field of view in a small subarea, splicing the reconstruction area to obtain the reconstruction result of the entire field of view. The splicing will not only bring a further errors, but also increase the calculating time; Furthermore, the speckle masking method assumes that the random phase fluctuation of the turbulent atmosphere is zero mean, which obviously does not meet the requirements of statistical characteristics within a short time scale $(<1$ second). Therefore, the time resolution of high-resolution solar reconstruction is usually about 10 seconds. Such limitations are difficult to meet the observation needs of rapidly changing objects, let alone the time resolution requirements of future large-aperture telescopes.

Based on the above analysis, this paper draws on the basic theoretical models of the speckle masking and Shift-Add, combined with the optical flow method in computer vision, and proposes a novelty high-resolution solar image reconstruction algorithm. This algorithm establishes a model of the relationship between intensity change and displacement field, and obtains the distortion displacement field of each frame of the speckle image relative to a reference image (RI). Then perform nonrigid pixel-by-pixel alignment for each frame of speckle images to correct distortion. Improve signal-to-noise ratio by ensemble averaging of aligned speckle images and use the phase of the averaged image as a phase estimate for the reconstructed object. The modulus of the object image is obtained by Wiener 
filtering on the average power spectrum of the aligned speckle images. A highresolution solar image reconstruction algorithm combining space and frequency domain is formed. We named the algorithm NASIR.

Section 2 of the paper introduces the principle and realization of phase reconstruction by NASIR. Section 3 applies the algorithm to reconstruct the actual observation data of NVST, and compares it with the classical methods. Section 4 discusses the advantages of NASIR and issues that need to be further studied. Our conclusions are given in Section 5 .

\section{The Principle and Implementation of NASIR}

In order to effectively improve the reconstruction quality of speckle imaging, NASIR corrects the distortion of each frame of speckle images through alignment, and ensemble averages the corrected (aligned) speckle images to improve the signal-to-noise ratio. The phase of the object image is estimated from the Fourier phase of the average image. The reconstruction of the object image modulus is obtained by speckle interferometry on the aligned speckle image sequence.

The above process involves key steps such as displacement field estimation, band-pass filtering, iterative alignment, and modulus reconstruction of the object image.

\subsection{Displacement Field Estimation Based on Intensity Changes}

In 1981, Horn and Schunck creatively connected the two-dimensional displacement field with the intensity, introduced the constraint equation, and obtained the basic algorithm for calculating the displacement information of the object (Horn and Schunck, 1981), which is called optical flow method. Based on the idea of optical flow method, we established the pixel displacement and intensity variation model of speckle image. Using this model, the phase distortion due to atmospheric turbulence is removed by reverse displacement correction.

For the convenience of analysis and calculation, we first use a polynomial to approximate the relationship between pixel intensity and pixel coordinates in a local region of the image. Taking the quadratic polynomial as an example, the intensity distribution of the local region in a speckle image can be approximately expressed as:

$$
f(\boldsymbol{x}) \approx \boldsymbol{x}^{T} \boldsymbol{A} \boldsymbol{x}+\boldsymbol{b}^{T} \boldsymbol{x}+c
$$

Where $\boldsymbol{x}$ is the coordinates of a local region in a speckle image, $f(\boldsymbol{x})$ is the pixel intensity at $\boldsymbol{x}, \boldsymbol{A}$ is a symmetric matrix, $\boldsymbol{b}$ is a displacement vector, and c is a scalar. These parameters can be estimated by weighted least square method.

Assume that the approximate expression formula of the corresponding region of the reference image RI (this article uses the ensemble average of the speckle images as the initial reference image) is:

$$
f_{1}(\boldsymbol{x}) \approx \boldsymbol{x}^{T} \boldsymbol{A}_{1} \boldsymbol{x}+\boldsymbol{b}_{1}{ }^{T} \boldsymbol{x}+c_{1}
$$


If the speckle image has an ideal translation $\boldsymbol{d}$ with respect to $\mathbf{R I}$ in this region, the relationship between the intensity of this area and the spatial coordinate of the speckle image can be expressed as:

$$
\begin{aligned}
f_{2}(\boldsymbol{x}) & =f_{1}(\boldsymbol{x}-\boldsymbol{d}) \\
& =(\boldsymbol{x}-\boldsymbol{d})^{T} \boldsymbol{A}_{1}(\boldsymbol{x}-\boldsymbol{d})+\boldsymbol{b}_{1}{ }^{T}(\boldsymbol{x}-\boldsymbol{d})+c_{1} \\
& =\boldsymbol{x} \boldsymbol{A}_{1} \boldsymbol{x}+\left(\boldsymbol{b}_{1}-2 \boldsymbol{A}_{1} \boldsymbol{d}\right)^{T} \boldsymbol{x}+\boldsymbol{d}^{T} \boldsymbol{A}_{1} \boldsymbol{d}-\boldsymbol{b}_{1}{ }^{T} \boldsymbol{d}+c_{1} \\
& =\boldsymbol{x}^{T} \boldsymbol{A}_{2} \boldsymbol{x}+\boldsymbol{b}_{2}{ }^{T} \boldsymbol{x}+c_{2}
\end{aligned}
$$

By comparing the coefficients of $f_{1}(\boldsymbol{x})$ and $f_{2}(\boldsymbol{x})$, we can get:

$$
\begin{aligned}
\boldsymbol{A}_{2} & =\boldsymbol{A}_{1} \\
\boldsymbol{b}_{2} & =\boldsymbol{b}_{1}-2 \boldsymbol{A}_{1} \boldsymbol{d} \\
c_{2} & =\boldsymbol{d}^{T} \boldsymbol{A}_{1} \boldsymbol{d}-\boldsymbol{b}_{1}^{T} \boldsymbol{d}+c_{1}
\end{aligned}
$$

In the non-singular case of $\boldsymbol{A}_{1}$, we can obtain the translation $\boldsymbol{d}$ through equation (5):

$$
\begin{gathered}
2 \boldsymbol{A}_{1} \boldsymbol{d}=-\left(\boldsymbol{b}_{2}-\boldsymbol{b}_{1}\right) \\
\boldsymbol{d}=(1 / 2) \boldsymbol{A}_{1}^{-1}\left(\boldsymbol{b}_{2}-\boldsymbol{b}_{1}\right)
\end{gathered}
$$

So we established a computational model between pixel intensity and displacement under ideal translation. In order to describe the projection of the distortion on the image more accurately, a more reasonable calculation model of the displacement field can be obtained through optimization strategies such as the affine transformation of the displacement field and the weighting of neighborhood pixels (Farnebäck, 2003). The specific method is as follows: for the projection of the general three-dimensional motion on the image plane, we use the affine transformation model to express the displacement field as:

$$
\begin{aligned}
& \boldsymbol{d}_{x}(x, y)=a_{1}+a_{2} x+a_{3} y+a_{7} x^{2}+a_{8} x y \\
& \boldsymbol{d}_{y}(x, y)=a_{4}+a_{5} x+a_{6} y+a_{7} x y+a_{8} y^{2}
\end{aligned}
$$

Written in matrix form:

$$
\boldsymbol{d}=\boldsymbol{S P}
$$

among them,

$$
\begin{gathered}
\boldsymbol{S}=\left(\begin{array}{cccccccc}
1 & x & y & 0 & 0 & 0 & x^{2} & x y \\
0 & 0 & 0 & 1 & x & y & x y & y^{2}
\end{array}\right) \\
\boldsymbol{P}=\left(\begin{array}{llllllll}
a_{1} & a_{2} & a_{3} & a_{4} & a_{5} & a_{6} & a_{7} & a_{8}
\end{array}\right)
\end{gathered}
$$


then:

$$
\boldsymbol{P}=\left(\sum_{i} w_{i} \boldsymbol{S}_{i}^{T} \boldsymbol{A}_{i}^{T} \boldsymbol{A}_{i} \boldsymbol{S}_{i}\right)^{-1} \sum_{i}\left(w_{i} \boldsymbol{S}_{i}^{T} \boldsymbol{A}_{i}^{T} \Delta \boldsymbol{b}_{i}\right)
$$

Where $i$ is the pixels in the neighborhood, and $w_{i}$ is the weights of the pixel $i$. Solving for $\boldsymbol{P}$, and then by formula(10), the displacement vector $\boldsymbol{d}$ of each pixel of the speckle image relative to $\mathbf{R I}$ can be obtained. By performing reverse displacement and interpolation of each pixel in each speckle image according to $\boldsymbol{d}$, the speckle image sequence can be aligned with RI.

\subsection{Band-pass Filtering}

Since the average atmospheric transmittance has a significant low-pass characteristic, if the original speckle images is directly used to estimate and correct the displacement field, it is essentially correcting the displacement characterized by the low-frequency structure, which is not conducive to effective reconstruction of the mid- and high-frequency phase. For this reason, based on the theoretical speckle interference transfer function(sitf) (Korff, 1973) and telescope optical transfer function (Otf) (yuan Xiang, Liu, and yu Jin, 2016), we constructed the band-pass filter $\boldsymbol{H}_{b p}(\boldsymbol{u})$ shown in equation (14) to perform band-pass filtering on the original speckle images to enhance the mid- and high- frequency features. Bandpass filters can also effectively suppress noise outside the diffraction limit.The speckle images after band-pass filtering is more conducive to alignment and phase reconstruction.

$$
\boldsymbol{H}_{b p}(\boldsymbol{u})=\frac{\operatorname{Otf}(\boldsymbol{u})}{\operatorname{sitf}(\boldsymbol{u})+\boldsymbol{S}_{n}}
$$

Where $\boldsymbol{S}_{n}$ is the noise power spectrum estimated by the speckle image sequence, and $\boldsymbol{u}$ is the spatial frequency.

\subsection{Reference Image Update and Iterative Alignment}

The estimation of distortion displacement requires an image with a higher signalto-noise ratio as a reference image $\mathbf{R I}$ (described in 2.1). The initial $\mathbf{R I}$ is obtained from the ensemble average of the original speckle image sequence filtered by the band-pass filter of equation (14). After the speckle image sequence is aligned with RI, the phase of the ensemble average image is corrected and the signal-to-noise ratio is improved. At this time, NASIR updates RI with the newer ensemble average image, and perform the displacement field estimation and alignment again.

After several iterations of alignment and update, the quality of $\mathbf{R I}$ is continuously improved, and the phase of speckle images is further restored. Then the phase of the ensemble average image will be used as an estimate of the phase of the object image. 


\subsection{Modulus Reconstruction Based on Speckle Interferometry}

The basic idea of speckle interferometry is that when the exposure time is less than a certain threshold, the speckle images contains more information than the long-exposure image. The power spectrum of multiple consecutive short exposure speckle images can be time-averaged to restore the power spectrum of the object image (Labeyrie, 1970).

In actual observations, speckle images often contain a variety of random noises. So, NASIR uses the Wiener filter (15) method to reconstruct the modulus of object image :

$$
|\boldsymbol{O}(\boldsymbol{u})|=\sqrt{\frac{<\left|\boldsymbol{I}_{i}(\boldsymbol{u})\right|^{2}>}{\operatorname{sift}(\boldsymbol{u})+\boldsymbol{S}_{n}}} \cdot \operatorname{Otf}(\boldsymbol{u})
$$

Where $|\boldsymbol{O}(\boldsymbol{u})|$ is the modulus of the object image, $\left\langle\left|\boldsymbol{I}_{i}(\boldsymbol{u})\right|^{2}\right\rangle$ is the ensemble average of the power spectrum of the speckle images, $\boldsymbol{S}_{n}$ is the noise power spectrum estimated from the speckle image sequence, sift $(\boldsymbol{u})$ is calculated based on the atmospheric seeing (Korff, 1973). Different from speckle masking, NASIR does not directly use the power spectrum of the original speckle image, but uses the power spectrum of the iteratively aligned speckle image sequence to calculate $\left\langle\left|\boldsymbol{I}_{i}(\boldsymbol{u})\right|^{2}>\right.$, and then obtain $|\boldsymbol{O}(\boldsymbol{u})|$ by equation (15). Since the aligned speckle images have the mid- and high-frequency phase corrected, their ensemble-averaged images contain richer mid- and high-frequency energy, which can further improve the contrast of detailed structures in the reconstructed images. This can be seen from the reconstruction results in section 3.2.

\section{Reconstruction Results and Analysis}

We use actual observation data from NVST to test the reconstruction performance of NASIR and compare with correlated Shift-Add (Shift-Add based on autocorrelation) and speckle masking algorithms.

NVST is the largest ground-based vacuum solar telescope in China, and it was put into use in 2010. The NVST has a clear aperture of $985 \mathrm{~mm}$ and is mainly used for high-resolution imaging observations of the solar photosphere, chromosphere, and solar spectrum observations (Liu et al., 2014). The main instruments of NVST include high-resolution multi-channel imaging systems (Xu et al., 2013) and high-dispersion multi-wavelength spectrometers(Wang et al., 2013).

NVST data products are divided into three levels: Level 0 is the original observation data; Level 1 is based on lucky imaging, corrected by dark current and flat field, and reconstructed by the Shift-Add; Level 1+ is reconstructed by speckle masking, which can produce reconstruction results close to the diffraction limit(Yan et al., 2020).

The experimental data set in this paper is derived from the Level 0 data of the NVST photosphere ( $\mathrm{TiO}$, center wavelength $705.8 \mathrm{~nm}$, bandwidth $1 \mathrm{~nm}$ ) and chromosphere $(\mathrm{H} \alpha$, center wavelength $656.28 \pm 0.4 \mathrm{~nm}$, bandwidth $0.025 \mathrm{~nm})$ 
Table 1. Speckle images high-resolution reconstruction test data set:

\begin{tabular}{ccccr}
\hline Data set No. & Band & Observation time & Active area & $\boldsymbol{R}_{0}(\mathrm{~cm})$ \\
\hline 1 & $\mathrm{TiO}$ & $2013-08-01$ UT 03:44:53 & 11801 & 10.29 \\
2 & $\mathrm{H} \alpha$ & $2019-10-04$ UT 02:59:05 & 12749 & 10.78 \\
3 & $\mathrm{TiO}$ & $2015-08-21$ UT 10:01:57 & 12403 & 5.88 \\
4 & $\mathrm{TiO}$ & $2019-04-11$ UT 08:43:27 & 12738 & 4.09 \\
\hline
\end{tabular}

under different seeing degrees from 2013 to 2019. Each set of experimental data contains 100 short-exposure speckle images (Table 1). The seeing parameter $\boldsymbol{R}_{0}$ is calculated from the spectral ratio method(von der Lühe, 1984).

\subsection{Analysis of Reconstruction Quality When Seeing is Good}

Figure 1. Shows the reconstruction results of NVST TiO observation data (data set No.1 in Table 1) using correlation Shift-Add, speckle masking and NASIR, respectively. Figure 2 is the power spectrum curve of the image reconstructed by the three algorithms.

As can be seen from Figure 1 and Figure 2, the correlation Shift-Add reconstructed images have a certain improvement in visual effects, but the highfrequency information is still insufficient (Figure 1(b)).

The reconstruction results of the speckle masking (Figure 1(c)) and NASIR (Figure 1(d)) are significantly better than the correlation Shift-Add in terms of overall visual quality and details (granule outlines, bright point and penumbral filaments of sunspot, etc.).

The power spectrum curve of the reconstructed image of the correlation Shift$A d d$ is cut off at about 0.25 arcsec, while the power spectrum curve of the speckle masking and the NASIR reconstructed image has a spatial cutoff frequency below 0.1 arcsec.

To further reveal the performance of NASIR, we use the following quantitative metrics to compare and analyze the reconstruction results of speckle masking and NASIR:

(1) Image correlation coefficient $(r)$ : The correlation coefficient is used for comparison the degree of linear correlation between reconstructed images, which is defined as:

$$
r(\boldsymbol{X}, \boldsymbol{Y})=\frac{\operatorname{Cov}(\boldsymbol{X}, \boldsymbol{Y})}{\sqrt{\operatorname{Var}[\boldsymbol{X}] \cdot \operatorname{Var}[\boldsymbol{Y}]}}
$$

Here, $\operatorname{Cov}(\boldsymbol{X}, \boldsymbol{Y})$ is the covariance of the pixel intensity of the two images $\boldsymbol{X}$ and $\boldsymbol{Y}, \operatorname{Var}[\boldsymbol{X}]$ and $\operatorname{Var}[\boldsymbol{Y}]$ are the variances of $\boldsymbol{X}$ and $\boldsymbol{Y}$ respectively.

(2) Structural SIMilarity (SSIM) (Wang et al., 2004): SSIM is an index that measures the similarity of two images. Given two images $\boldsymbol{X}$ and $\boldsymbol{Y}$, their structural similarity can be found in the following way:

$$
\operatorname{SSIM}(\boldsymbol{X}, \boldsymbol{Y})=\frac{\left(2 \mu_{x} \mu_{y}+c_{1}\right) \cdot\left(2 \sigma_{x y}+c_{2}\right)}{\left(\mu_{x}^{2}+\mu_{y}^{2}+c_{1}\right) \cdot\left(\sigma_{x}^{2}+\sigma_{y}^{2}+c_{2}\right)}
$$



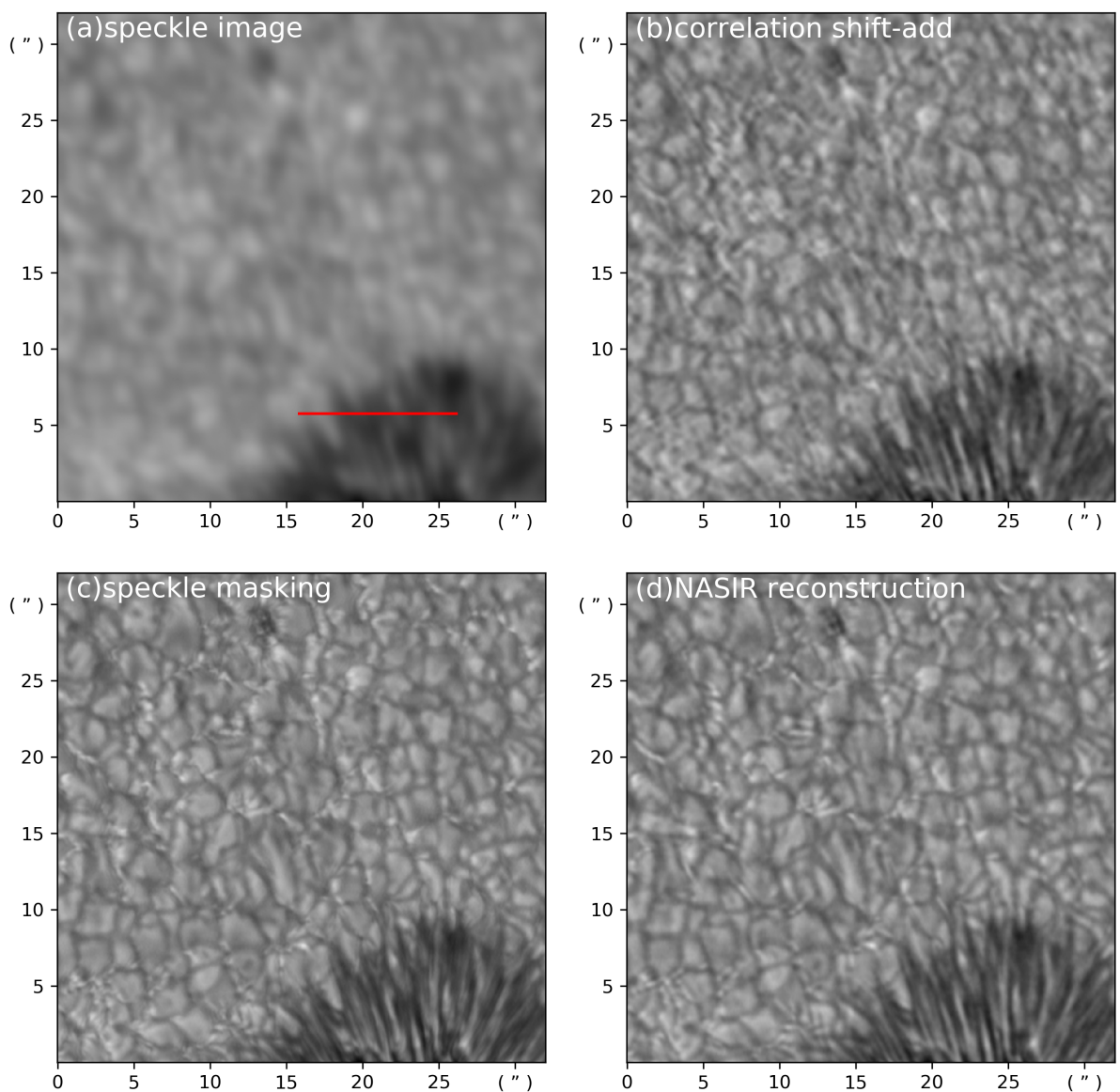

Figure 1. Reconstruction results of the NVST TiO observations with the active area 11801, 2013-08-01T:03:44:53. (a) A frame in a sequence of speckle images. (b) Reconstruction by correlation Shift-Add. (c) Reconstruction by speckle masking. (d) NASIR reconstruction.

Where $\mu_{x}$ is the mean of $\boldsymbol{X}, \mu_{y}$ is the mean of $\boldsymbol{Y}, \sigma_{x}$ is the variance of $\boldsymbol{X}, \sigma_{y}$ is the variance of $\boldsymbol{Y}$, and $\sigma_{x y}$ is the covariance between $\boldsymbol{X}$ and $\boldsymbol{Y} . c_{1}=\left(k_{1} L\right)$, $c_{2}=\left(k_{2} L\right)$ are constants used to maintain stability. $L$ is the range level of pixel values. $k_{1}=0.03, k_{2}=0.03$. The range of structural similarity is -1 to 1 . When the two images are exactly same, the value of SSIM is equal to 1.

The structural similarity index defines structural information from the perspective of image composition as being independent of brightness and contrast, reflecting the properties of the object structure in the scene, and modeling distortion as a combination of three different factors, brightness, contrast, and structure. The mean is used as an estimate of brightness, standard deviation is used as an estimate of contrast, and covariance is used as a measure of structural similarity.

(3) Coefficient of Variation of Intensity Profile $(C V o I P)$ : We use the coefficient of variation of the intensity profile of reconstructed image, which is 


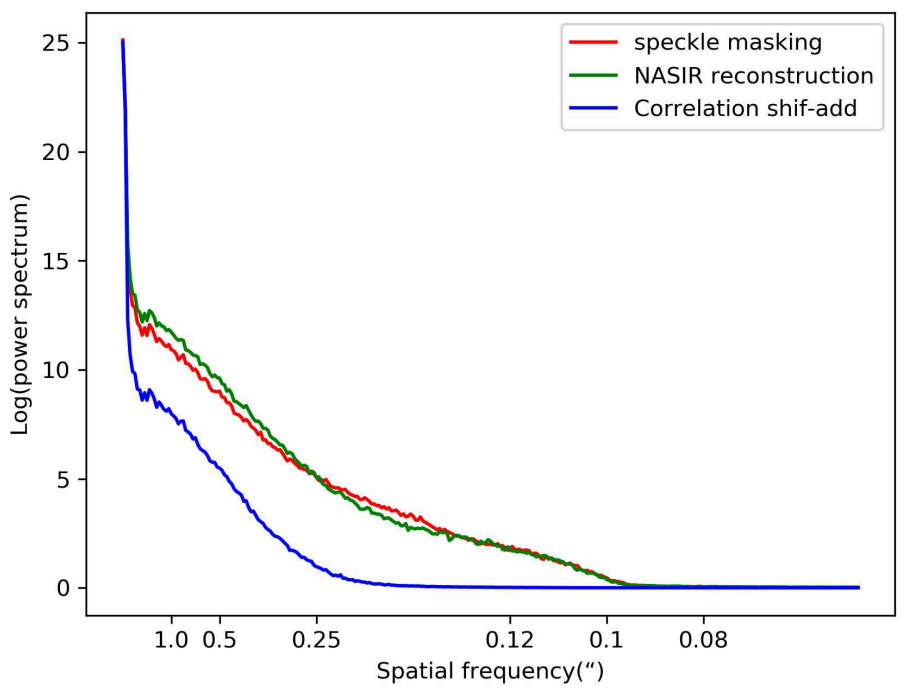

Figure 2. Power spectrum curves of images reconstructed by three methods

Table 2. Quantitative comparison of the reconstruction results of the four groups of test data (Table 1).

\begin{tabular}{|c|c|c|c|c|}
\hline \multirow[t]{2}{*}{ Data set No. } & \multirow[t]{2}{*}{$r$} & \multirow[t]{2}{*}{ SSIM } & \multicolumn{2}{|l|}{ CVoIP } \\
\hline & & & speckle masking & NASIR \\
\hline 1 & 0.9802 & 0.9561 & 0.5022 & 0.5359 \\
\hline 2 & 0.9489 & 0.8887 & 0.3580 & 0.4078 \\
\hline 3 & 0.9129 & 0.8154 & 0.3636 & 0.4911 \\
\hline 4 & 0.5182 & 0.5417 & 0.4608 & 0.4795 \\
\hline
\end{tabular}

the ratio of the standard deviation of the intensity profile to its mean, to measure the difference in image contrast.

A quantitative comparison results of speckle masking with NASIR are shown in Table 2, indicating that the reconstructed images have high correlation and structural similarity. However, the contrast of NASIR is slightly better than that of speckle masking, and their intensity profile (pixel intensities corresponding to the red line in Figure 1(a) ) is compared as shown in Figure 3.

Therefore, from the visual quality (Figure 1), the consistency of the power spectrum curve (Figure 2), and the quantitative comparison index of Table 2, it can be seen that NASIR has achieved a reconstruction quality equivalent to that of the speckle masking method when the seeing is good.

The reconstruction results of the $\mathrm{H} \alpha$-band data further verify the effectiveness of the NASIR for high-resolution reconstruction of the speckle images when the seeing is better. Figure 4 shows the reconstructed images and power spectrum curves of the three algorithms for data set 2 . The quantitative comparison result 


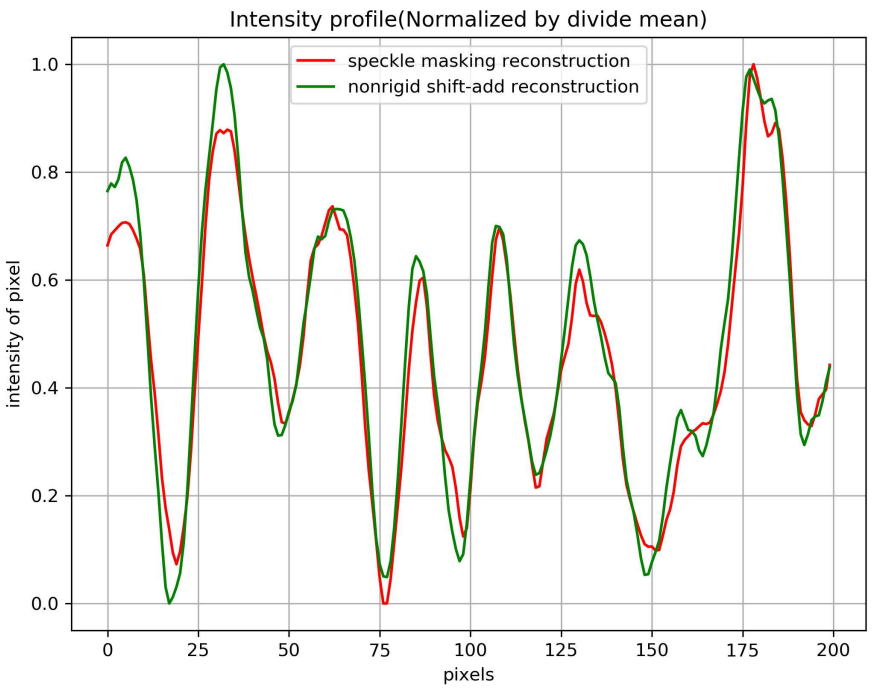

Figure 3. Comparison of the intensity profile (pixels under the red line in Figure 1.(a)) between the reconstruction results of speckle masking and NASIR. red: speckle masking; green: NASIR

is the second row in Table 2, and the intensity profile curve is shown in Figure 5. The reconstructed image of the NASIR and the reconstructed image of the speckle masking have a higher structural similarity, and the contrast of the reconstructed image of NASIR is improved to a certain extent.

\subsection{Comparison of Reconstruction Performance with Slightly Poor Seeing}

As the seeing decreases, the signal-to-noise ratio of the observed data becomes lower, and the recursive accumulation error of the high-frequency phase of speckle masking becomes more serious, resulting in spurious structures in the reconstructed image, while the NASIR algorithm shows stronger robustness. Figure 6 shows the comparison of the reconstruction results of data set $3\left(\boldsymbol{R}_{0}=5.88 \mathrm{~cm}\right)$. It can be seen that the phase recursive error of speckle masking begins to produce crisscrossed and twisted structures, while the reconstruction results of NASIR can better maintain the characteristics of granules. The reconstruction results of the two algorithms still have high correlation and structural similarity, but the CVoIP values reflect the higher contrast of the reconstructed images from NASIR. The third row of Table 2 gives a comparison of quantitative indicators.

With the further decrease of seeing, the phase recurrence error of speckle masking seriously affects the reconstruction quality, and the whole reconstructed image has been destroyed by the pseudo-structure, while the NASIR can still effectively reconstruct the low-frequency structure. Figure 7 shows the comparison of the reconstruction results of data set $4\left(\boldsymbol{R}_{0}=4.09 \mathrm{~cm}\right)$ in Table 1 . Figure 8 presents their power spectral curves and intensity profiles. The correlation 
Hui Liu et al.
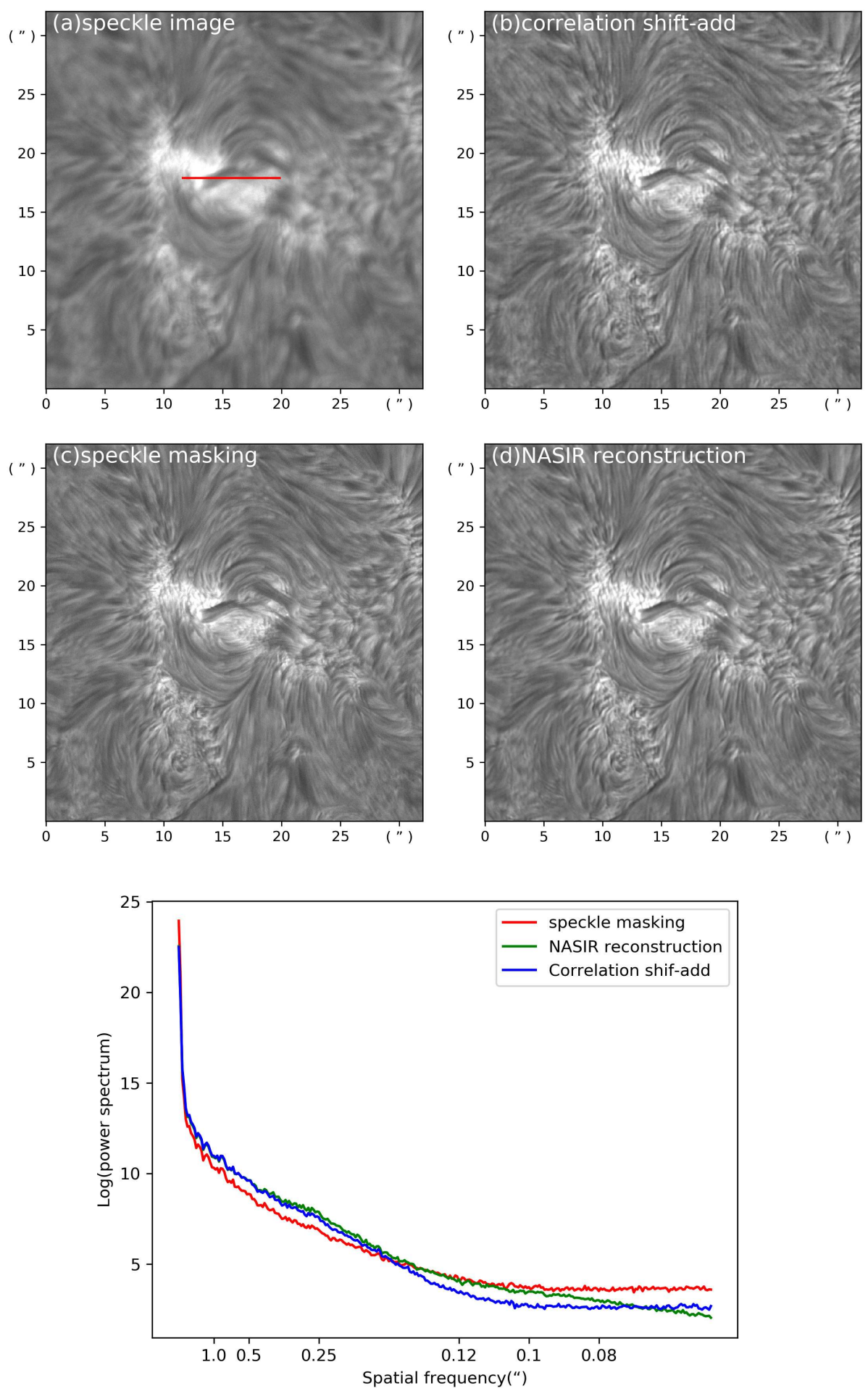

Figure 4. Reconstruction results and power spectrum of NVST H $\alpha$ 2019-10-04T02:59:05 observation (active area 12749). 


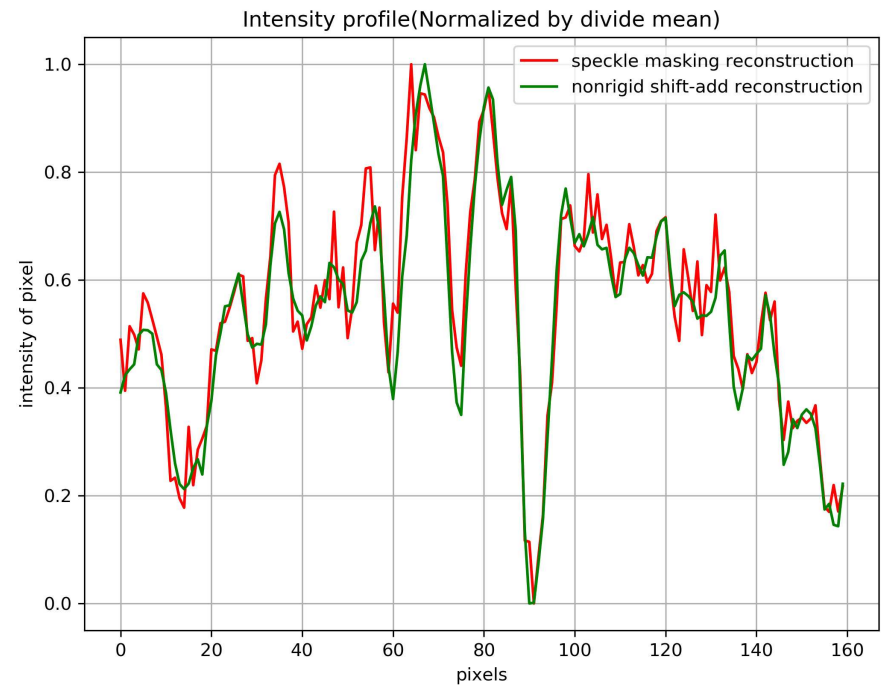

Figure 5. Intensity contour comparison of $\mathrm{H} \alpha$-band reconstruction results
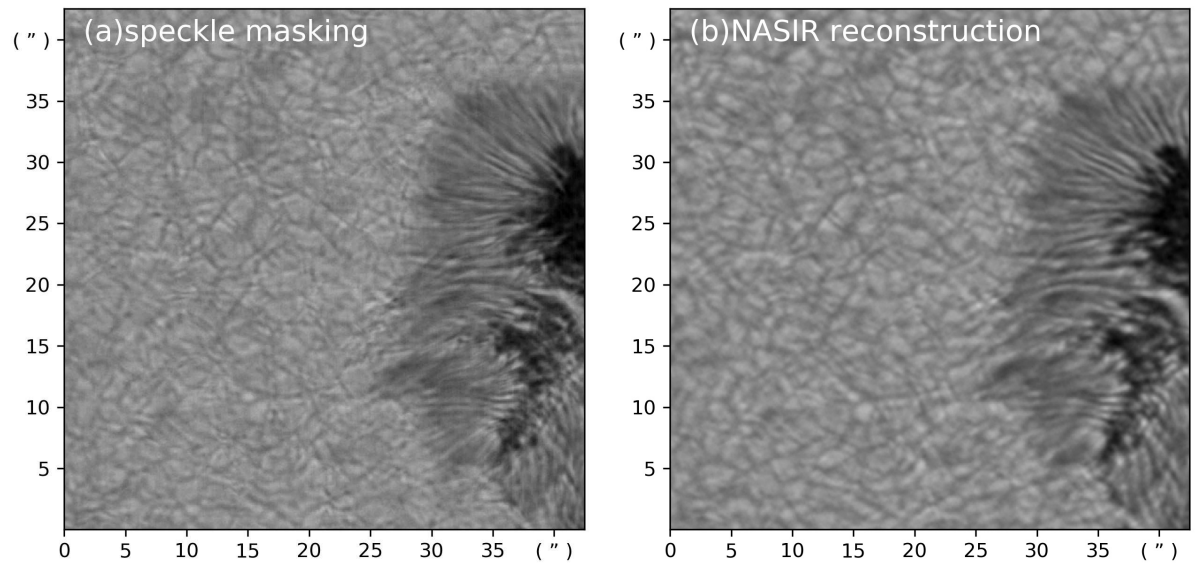

Figure 6. Comparison of reconstruction results when Fried constant $\boldsymbol{R}_{0}=5.88 \mathrm{~cm}$, (a) speckle masking (b) NASIR.

coefficient and SSIM of the reconstruction results of the two algorithms are significantly reduced, indicating that there is a significant difference between the reconstructed images. From the results in Figure 7 and Figure 8, The reconstruction results of NASIR have more abundant mid-high frequency energy and more prominent contrast, so the reconstruction performance of NASIR is more stable when the seeing is slightly worse. The corresponding quantitative comparisons are shown in the fourth row of Table 2. 

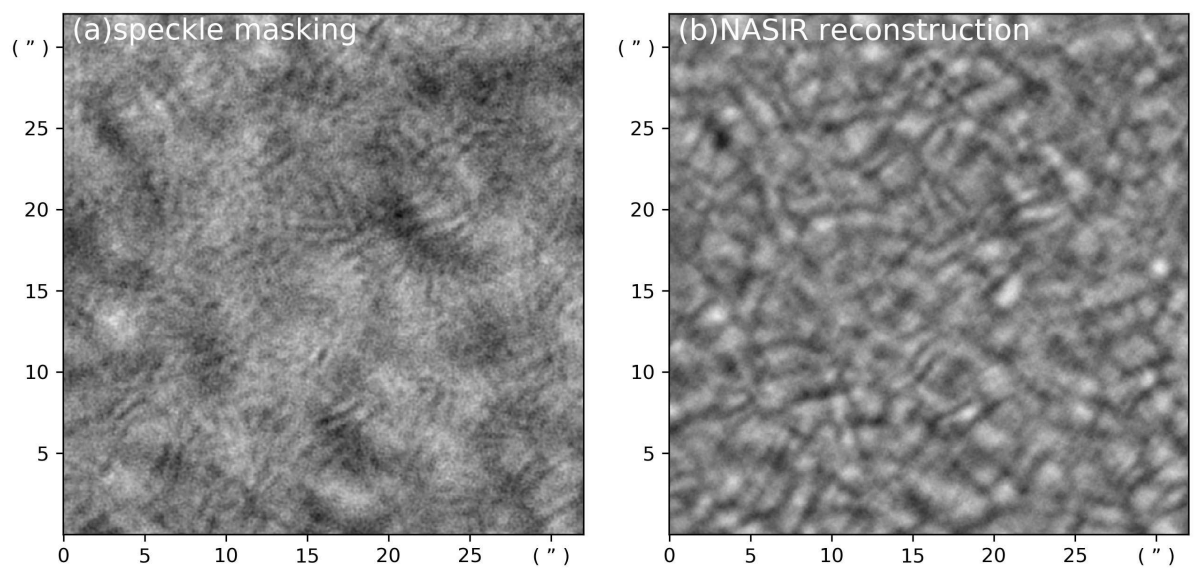

Figure 7. Comparison of reconstruction results when Fried constant $\boldsymbol{R}_{0}=4.09 \mathrm{~cm}$, (a) speckle masking, (b) NASIR.
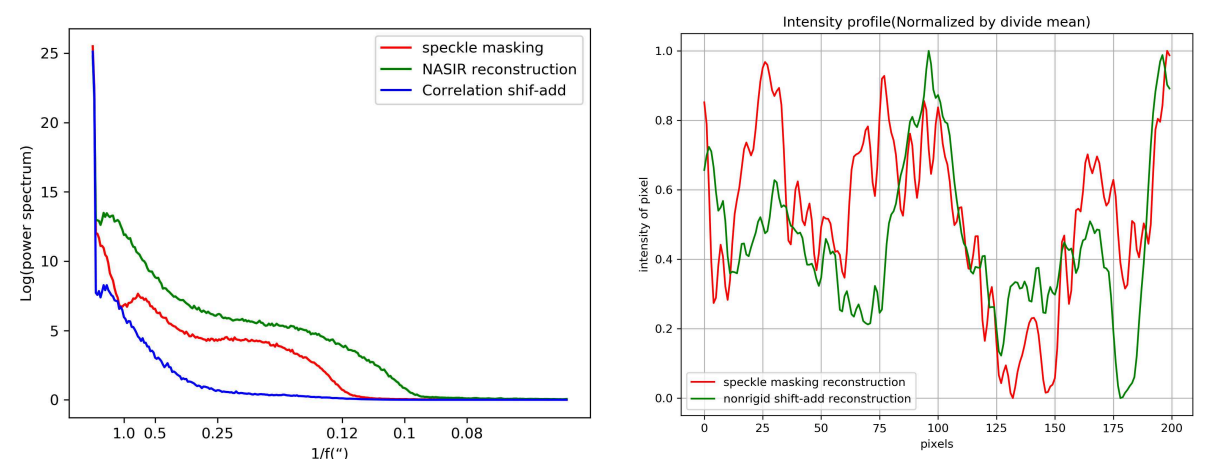

Figure 8. Comparison of the power spectrum curve (left) and the intensity distribution (right) of the reconstruction results when $\boldsymbol{R}_{0}=4.09 \mathrm{~cm}$ (Figure 7 ).

\subsection{Computational Time-consuming}

NASIR is a spatially parallel algorithm that avoids the multipath phase recursion, block reconstruction and stitching, so its average computation time is about $48.7 \%$ of speckle masking.

\section{Discussion}

The core idea of NASIR is to directly reconstruct the object phase through the spatial distortion correction of the speckle image sequence. From the experiments and analysis in Section 3, it can be seen that NASIR shows advantages and potential in many aspects. However, NASIR also has some specific issues that require further refinement and improvement. 


\subsection{Advantages of NASIR}

Compared with speckle masking, the current classical method for high-resolution reconstruction of solar images, NASIR has the following advantages:

No spurious structures are produced in the reconstruction results. Because NASIR estimates the displacement field for each pixel of the full field of view in parallel, it is immune to recursive error accumulation. This can be seen from Figures 6 and 7 .

Bandpass filtering of the original speckle images enhances the effective information within the diffraction limit, enabling NASIR to more efficiently reconstruct the phases of mid- and high-frequency structures. This can be seen from the power spectrum curves in Figures 2 and 4.

NASIR performs phase correction through pixel-by-pixel nonlinear alignment, which can directly reconstruct the full field of view at one time, without subregion segmentation and reconstruction like speckle masking, so there will be no stitching errors.

NASIR avoids the computation of high-order statistics and complex multipath phase recurrence, and the computation time is only about half that of speckle masking.

\subsection{Further Improvements to NASIR}

While achieving successful experiments, NASIR deserves further optimization and improvement in the following areas:

(1) Optimization of displacement field-intensity distribution model. Considering the convenience of analysis and calculation, NASIR adopts polynomial expansion to approximate the intensity distribution of the image area. This may differ from physical models of true intensity changes caused by atmospheric turbulence. Therefore, establishing a speckle image intensity distributiondisplacement representation model that is more in line with physical properties is one of the issues worthy of further study.

(2) Adaptive selection of neighborhood size. NASIR uses the intensity distribution model of a certain neighborhood as a feature descriptor, and estimates the displacement through the matching between feature descriptors. A larger neighborhood size can improve the stability of the matching, but it will produce a wider range of smoothing effects and reduce the reconstruction quality of highfrequency structures. On the contrary, a small neighborhood size can preserve high-frequency details, but in the case of poor seeing and strong noise, it is easy to cause mismatch and reduce the accuracy of displacement estimation. Therefore, a more reasonable neighborhood size should be adaptively selected according to the atmospheric coherence length to obtain better alignment performance.

(3) Design and implementation of a bandpass filter based on seeing. NASIR uses bandpass filtered speckle images for alignment, which is essentially an attempt to phase correction based on object structure within the diffraction limit. Therefore, the design of bandpass filters based on seeing is also one of the key issues that need to be further considered for phase reconstruction. 
(4) Iterative strategy for alignment operations and reference image updates. Non-rigid alignment based on speckle imaging requires a reference image. Iterative improvement of reference image quality is important for phase reconstruction. The number of iterations of NASIR for the reference image is selected between $3 \sim 5$ times according to $\boldsymbol{R}_{0}$, so it is necessary to make more rational choices and judgments for iteration and convergence.

\section{Conclusion}

NASIR is a novel high-resolution reconstruction method for speckle imaging that combines classical physical statistics and computer vision techniques. Reconstruction results from ground-based telescope observations demonstrate the strengths and prospects of NASIR in several ways:

NASIR is more robust. When the seeing is good, the reconstruction quality of NASIR is close to that of the speckle masking, and as the seeing degree decreases, the reconstruction quality of NASIR is better than that of the speckle masking.

The application of NASIR is more flexible and convenient. NASIR can directly reconstruct the full field of view without sub-block reconstruction and stitching, avoiding stitching errors and discontinuities between stitched sub-blocks.

NASIR is more computationally efficient. NASIR does not perform phase recursion and also avoids block reconstruction/stitching operations. Compared with the speckle masking, the average computation time is reduced by nearly $50 \%$.

In summary, NASIR provides a more convenient and efficient new method for high-resolution solar image reconstruction. With the further research and optimization of NASIR, its theoretical explanation will be more perfect, and its application will also promote the development of ground-based observation data processing.

Acknowledgments We would like to thank the NVST team for high cadence data support. This work is sponsored by the National Natural Science Foundation of China (NSFC) under the grant numbers (11873027, U2031140, 12073077, 11833010, 11973088), and West Light Foundation of the Chinese Academy of Sciences (Y9XB01A, Y9XB019).

\section{References}

Bates, R.H.T., Cady, F.M.: 1980, Towards true imaging by wideband speckle interferometry. Optics Communications 32, 365. DOI. https://www.sciencedirect.com/science/article/pii/ 0030401880902618.

Cao, W., Gorceix, N., Coulter, R., Ahn, K., Rimmele, T., Goode, P.R.: 2010, Scientific instrumentation for the $1.6 \mathrm{~m}$ new solar telescope in big bear. Astronomische Nachrichten $\mathbf{3 3 1}$, 636.

Farnebäck, G.: 2003, Two-frame motion estimation based on polynomial expansion. In: Scandinavian conference on Image analysis, 363. Springer.

Horn, B.K., Schunck, B.G.: 1981, Determining optical flow. Artificial intelligence 17, 185.

Huo, Z.-X., Zhou, J.-F.: 2010, Methods of astronomical image reconstruction from speckle image. Progress in Astronomy 28, 72. ADS. 
Jin, Z., Lin, J., Liu, Z.: 2008, High-resolution image reconstruction technique applied to the optical testing of ground-based astronomical telescopes. In: Ground-based and Airborne Telescopes II 7012, 70122U. International Society for Optics and Photonics.

Korff, D.: 1973, Analysis of a method for obtaining near-diffraction-limited information in the presence of atmospheric turbulence. JOSA 63, 971.

Labeyrie, A.: 1970, Attainment of Diffraction Limited Resolution in Large Telescopes by Fourier Analysing Speckle Patterns in Star Images. Astrophys. J. 6

Liu, Z., Qui, Y., Ke, L., Lu, R.: 1999, Phase recovery methods in speckle masking and image reconstruction experiment. Acta Optica Sinica 19, 935. ADS.

Liu, Z., Xu, J., Gu, B.-Z., Wang, S., You, J.-Q., Shen, L.-X., Lu, R.-W., Jin, Z.-Y., Chen, L.-F., Lou, K., et al.: 2014, New vacuum solar telescope and observations with high resolution. Research in Astronomy and Astrophysics 14, 705.

Lohmann, A.W., Weigelt, G., Wirnitzer, B.: 1983, Speckle masking in astronomy: triple correlation theory and applications. Applied Optics 22, 4028.

Qiu, Y.H., Liu, Z., Lu, R.W., Lou, K.: 2001, A New Method for Astronomical Image Reconstruction in Spatial Region: Iterative Shift-and-Add Technique. Acta Optica Sinica.

von der Lühe, O.: 1984, Estimating Frieds parameter from a time series of an arbitrary resolved object imaged through atmospheric turbulence. JOSA A 1, 510.

Wang, R., Xu, Z., Jin, Z.-Y., Li, Z., Fu, Y., Liu, Z.: 2013, The first observation and data reduction of the Multi-wavelength Spectrometer on the New Vacuum Solar Telescope. Research in Astronomy and Astrophysics 13, 1240.

Wang, Z., Bovik, A.C., Sheikh, H.R., Simoncelli, E.P.: 2004, Image quality assessment: from error visibility to structural similarity. IEEE transactions on image processing 13, 600.

Xu, Z., Jin, Z.Y., Xu, F.Y., Liu, Z., et al.: 2013, Primary observations of solar filaments using the multi-channel imaging system of the New Vacuum Solar Telescope. Proceedings of the International Astronomical Union 8, 117.

Yan, X., Liu, Z., Zhang, J., Xu, Z.: 2020, Research progress based on observations of the New Vacuum Solar Telescope. Science China Technological Sciences 63, 1656.

Xiang, Y.-y., Liu, Z., Jin, Z.-y.: 2016, High resolution reconstruction of solar prominence images observed by the New Vacuum Solar Telescope. New Astronomy 49, 8. DOI. https://www. sciencedirect.com/science/article/pii/S1384107616300197. 